\title{
Review
}

\section{All motors have to decide is what to do with the DNA that is given them}

\begin{abstract}
DNA translocases are a diverse group of molecular motors responsible for a wide variety of cellular functions. The goal of this review is to identify common aspects in the mechanisms for how these enzymes couple the binding and hydrolysis of ATP to their movement along DNA. Not surprisingly, the shared structural components contained within the catalytic domains of several of these motors appear to give rise to common aspects of DNA translocation. Perhaps more interesting, however, are the differences between the families of translocases and the potential associated implications both for the functions of the members of these families and for the evolution of these families. However, as there are few translocases for which complete characterizations of the mechanisms of DNA binding, DNA translocation, and DNA-stimulated ATPase have been completed, it is difficult to form many inferences. We therefore hope that this review motivates the necessary further experimentation required for broader comparisons and conclusions.
\end{abstract}

Keywords: ATPase; molecular motor; translocase.

DOI 10.1515/bmc-2014-0017

Received June 17, 2014; accepted September 9, 2014

\section{Introduction}

The ability of proteins to move directionally along nucleic acids is fundamental to many key biological processes. Perhaps best known among these molecular motors are DNA and RNA helicases. The central function shared by these motors is the coupling of the binding and hydrolysis of ATP to the translocation along and/or unwinding

*Corresponding author: Christopher J. Fischer, Department of Physics and Astronomy, University of Kansas, 1082 Malott Hall, 1251 Wescoe Hall Dr., Lawrence, KS 66045, USA, e-mail: shark@ku.edu Koan Briggs: Department of Physics and Astronomy, University of Kansas, 1082 Malott Hall, 1251 Wescoe Hall Dr., Lawrence, KS 66045, USA of single-stranded nucleic acids and double-stranded nucleic acids (1-6). Other motors use their ability to translocate along double-stranded DNA (dsDNA) to reposition nucleosomes (7-9), to transport nucleic acids into or out of viral capsids $(10,11)$, or to process DNA for nuclease activity (12-18).

In studies of the single-stranded (ssDNA) and dsDNA translocation activity of motor proteins, several key questions present themselves.

Firstly, what is the kinematic mechanism of translocation? The main associated descriptions are the directionality of translocation, the macroscopic translocation rate, the kinetic step-size, the physical step-size, and the processivity of translocation (19-21). The directionality of translocation is described in terms of the intrinsic polarity of the DNA molecule itself. For example, a $3^{\prime}-5^{\prime}$ ssDNA motor translocates away from the $3^{\prime}$ end of the ssDNA and toward the $5^{\prime}$ end of the ssDNA. The directionality of dsDNA translocases is similarly specified based upon the polarity of their directional tracking along one of the strands in the duplex. The physical step-size of translocation is best considered to be the number of nucleotides or basepairs translocated by the motor for each ATP hydrolyzed. The physical (or mechanical) step-size is thus a constraint imposed by the structure of the motor itself and/or the geometry with which it binds to the DNA. The kinetic step-size, conversely, is the number of nucleotides or basepairs translocated by the motor between each rate-limiting step in its translocation cycle. Determinations of both step-sizes are required for a complete description of the mechanism of translocation. Finally, the processivity of translocation is the propensity of the motor to translocate further along the DNA rather than dissociate from it. It is usually expressed either as a ratio of the probability of forward motion to dissociation or in terms of the average number of nucleotides (or basepairs) translocated before dissocation.

Secondly, what is the efficiency of motor translocation? Efficiency is usually described in terms of the number of ATP molecules hydrolyzed per nucleotide (ssDNA) or basepair (dsDNA) translocated. Thus, it often provides an estimate of the physical step-size of translocation. 
Thirdly, what are the structural intermediates of the DNA:motor complex during the translocation reaction? For example, many motors are believed to form DNA loops during translocation (22-26). By considering motor efficiency, the distinction between kinetic and physical step-sizes of the translocation mechanism can be elucidated. Finally, what can we learn about the evolutionary pressures and common structure/function motifs from the mechanisms of action of these motors?

The goal of this review is to highlight recent research in this field. With a primary focus on the unifying characteristics of recently determined DNA translocation mechanisms and their associated implications for the structure/ function relationships intrinsic to this important class of enzymes.

\section{Experimental approaches and mechanistic models}

Given the importance of DNA translocation to such a wide variety of cellular function it is not surprising that a large number of assays have been developed to monitor it (Figure 1). Several of the most commonly used methods of monitoring the translocation of a motor protein are: a) measuring the arrival of the protein at a particular point along the DNA (19-24); b) detecting the displacement of obstacles from DNA by the translocating motor (25-27); c) DNA-dependent ATPase activity of the motor $(18,22$, 27-31); d) translocation-induced changes in the structure/ topology of the DNA $(32,33)$. While each approach has
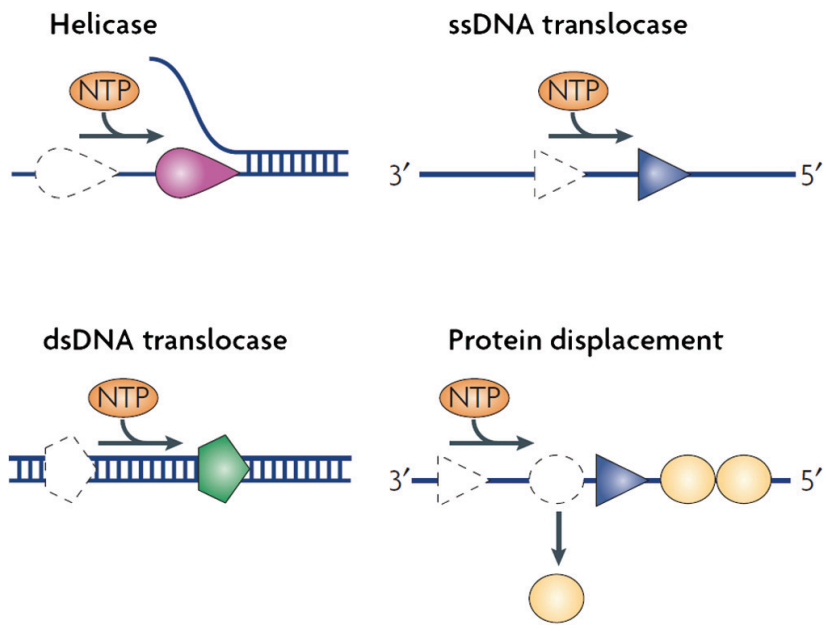

Figure 1 Four common modes of nucleic acids strand translocases. Image taken from ref. (5), with permission. advantages and disadvantages, addressing the key questions typically involves a combined approach of several of these techniques.

There are several common models for DNA translocation by molecular motors. According to the first model, commonly referred to as an inchworm or ratchet model (Figure 2) $(5,28,34-36)$ coordinated sequential alterations in the binding and release of the DNA by residues within the motor propels the motor along the DNA. A modified version of this model is the Brownian ratchet in which ATP binding by the motor weakens the motor's DNA binding affinity. This allows for a period of Brownian motion to occur before ATP hydrolysis again prompts DNA binding, albeit now with a bias toward net forward displacement of the motor along the DNA $(2,37,38)$. In contrast, a purely Brownian motor would have no tendency toward directionally biased translocation along the DNA. It follows that efficient coupling of ATP hydrolysis to translocation (i.e., the hydrolysis of few ATP molecules required per nucleotide or basepair of net translocation of the motor away from its starting point) is indicative of an inchworm model, whereas inefficient coupling of ATP hydrolysis to translocation (i.e., the hydrolysis of many ATP molecules required per nucleotide or basepair of net translocation of the motor away from its starting point) is indicative of a purely Brownian motor.

\section{DNA translocation by DNA helicases}

The fundamental distinction of DNA helicases is their ability to couple the binding and hydrolysis of nucleoside triphosphates to dsDNA unwinding $(5,6,39-41)$. From there several traits are used to further categorize DNA helicases. Unidirectional translocation of DNA helicases along ssDNA has been inferred from the results of several in vitro studies demonstrating that nearly all helicases show a preference for unwinding DNA substrates possessing either a $3^{\prime}$ or $5^{\prime}$ ssDNA tail flanking the dsDNA substrate $(6,39,41)$. Therefore, helicases preferring a $3^{\prime}$ tail are classified as $3^{\prime}-5^{\prime}$ whereas helicases preferring a $5^{\prime}$ tail are classified as $5^{\prime}-3^{\prime}$ helicases. Furthermore, helicases have been divided into six superfamilies (SF1-SF6) based on conserved sequence motifs (41-43), with a majority belonging to SF1 and SF2 $(43,42)$. SF1 and SF2 helicases function primarily as monomers or dimers whereas the members of SF3 through SF6 are typically hexameric rings. Most recent mechanistic studies of DNA translocation by helicases have focused on members of SF1 and SF2 and, accordingly, they will represent most of the discussion in 


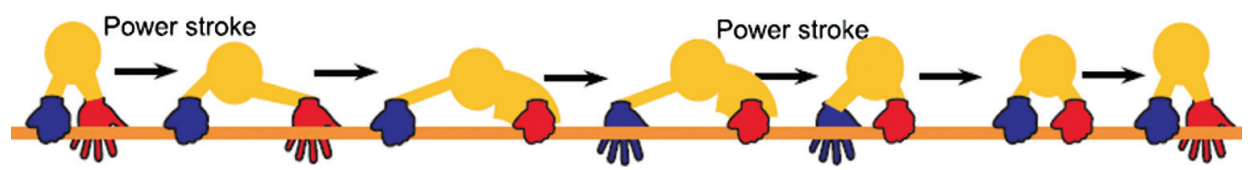

Figure 2 This cartoon representation of the inchworm translocation mechanism clearly shows that the protein has at least one strong attachment to the polynucleotide track at any one time and the two binding sites (red and blue) maintain their order along the DNA. Image taken from ref. (4) with permission.

this section. A unifying characteristic of SF1 and SF2 helicases is a conserved core of protein domains similar to the homologous recombination protein RecA (41), several of these domains are also found in members of other superfamilies (41-43).

\section{SF1 family helicases}

The UvrD helicase from Escherichia coli is a $3^{\prime}-5^{\prime}$ SF1 DNA helicase involved in methyl-directed mismatch repair and nucleotide excision repair of DNA (44). Monomers of UvrD translocate from $3^{\prime}$ to $5^{\prime}$ along ssDNA (45) hydrolyzing 1 ATP molecule for each nucleotide translocated $(22,28)$. This tight coupling of ATP hydrolysis to translocation is consistent with an inchworm model of ssDNA translocation by UvrD and further suggests a physical step-size of one nucleotide for this process. However, the kinetic stepsize for ssDNA translocation by UvrD has been determined to be four to five nucleotides $(22,28,45)$, indicating that the rate-limiting process in the translocation mechanism occurs approximately every four or five nucleotides translocated (28).

This disparity between the physical and kinetic step-sizes of translocation can be reconciled by a non-uniform stepping mechanism $(22,28)$. Specifically, ssDNA translocation by UvrD is believed to comprise two processes. A fast process associated with the forward translocation of the motor one nucleotide along the ssDNA and a slow process, occurring after four or five repetitions of the fast process that is ratelimiting for the overall translocation reaction. It has further been suggested that this slow process is associated with the resolution of DNA loops created by the associated faster physical translocation steps (28). This would imply that the size of these loops is on the order of four or five nucleotides.

Analysis of ensemble measurements of ssDNA translocation by UvrD at saturated ATP resulted in an estimate of $(193 \pm 1) \mathrm{nt} / \mathrm{s}$ for the macroscopic rate of ssDNA translocation and an estimate that UvrD translocates (769 1 ) nucleotides on average before dissociating from the
ssDNA (28). Estimates of $193 \mathrm{nt} / \mathrm{s}$ and 1260 nucleotides for these parameters have been obtained from the analysis of the results of single-molecule measurements of UvrD translocation (46). Furthermore, the results of these single-molecule experiments suggest that the four to five nucleotides kinetic step-size for ssDNA translocation by UvrD cannot be attributed to molecular heterogeneity within the UvrD population (46); i.e., this kinetic stepsize cannot be explained by the presence of a distribution of translocation activities (rates, step-sizes, etc) for the UvrD enzymes. Indeed, the existence of such persistent molecular heterogeneity for the entire translocation process would be an example of static disorder $(47,48)$ and could result only if each enzyme were chemically or conformationally different over the time period of the experiment.

The PcrA helicase from Bacillus stearothermophilus is a $3^{\prime}-5^{\prime}$ SF1 DNA helicase involved in DNA repair pathways and the rolling circle replication of some plasmids within that organism $(49,50)$. Monomers of PcrA have been shown to translocate from $3^{\prime}$ to $5^{\prime}$ along ssDNA (24, 30, 51, 52) with a macroscopic rate between $50 \mathrm{nt} / \mathrm{s}$ (30) and over $200 \mathrm{nt} / \mathrm{s}$ (52) depending upon the solution conditions of the experiment. Although there has been no calculation of the processivity of ssDNA translocation by PcrA it can be safely argued that the enzyme will translocate more than $50 \mathrm{nt}$ on average before dissociating from the ssDNA (24, $30,53)$.

Interestingly the kinetic step-size for ssDNA translocation is independent of the solution conditions of the experiment and has an average value of $\sim 4$ nucleotides (52). However, just as for UvrD, PcrA hydrolyzes one molecule of ATP for each nucleotide it moves along the ssDNA (30) indicating that the physical step-size for ssDNA translocation is one nucleotide and thus smaller than the kinetic step-size. As before, this is consistent with a non-uniform inchworm model for ssDNA translocation by PcrA. Furthermore, it is tempting to conclude that the resolution of small ssDNA loops formed by PcrA is the rate-limiting process associated with the $\sim 4$ nucleotide kinetic step-size. An inchworm model for ssDNA translocation by PcrA is also consistent with the observation that 
the products of ATP hydrolysis associated with PcrA translocation along ssDNA are released in two steps, with $\mathrm{P}_{\mathrm{i}}$ released before ADP (54). Based on this result it has been suggested that the major transition in the structure of the PcrA:DNA complex, possibly associated with the powerstroke of the translocation mechanism (Figure 2), is associated with ADP release.

The Rep helicase from Escherichia coli is a $3^{\prime}-5^{\prime}$ SF1 DNA helicase that is involved in replication restart (55) and is required for replication of some bacteriophages (56). Monomers of Rep are capable of translocating from $3^{\prime}$ to $5^{\prime}$ along ssDNA with a macroscopic rate that varies with solution conditions (51). At saturating ATP concentrations Rep translocates at $(279 \pm 2) \mathrm{nt} / \mathrm{s}$ at low solution $\mathrm{NaCl}$ concentration and at $(298 \pm 2) \mathrm{nt} / \mathrm{s}$ at high solution $\mathrm{NaCl}$ concentration (51). As an increase in the solution salt concentration would likely decrease the affinity of ssDNA binding by Rep these results suggest an inverse relationship between DNA binding affinity and translocation rate.

Interestingly, during their translocation along ssDNA UvrD, Rep and PcrA all experience a slow dissociation upon reaching the end of the DNA $(22,28,45,51,52)$. Frequently this is associated with additional 'futile' ATP hydrolysis by the motor while it remains bound to the end of the DNA (i.e., ATP hydrolysis not coupled with forward translocation).

UvrD, PcrA, and Rep all contain a single ATP hydrolysis site and are capable of translocating along ssDNA as monomers. There are also SF1 helicase complexes that contain more than one ATP hydrolysis site (i.e., that contain more than one motor) because more than one complex member contains an ATP hydrolysis site (i.e., that is separately classified as a motor). A thoroughly studied example is the SF1 RecBCD DNA helicase from $E$. coli. RecBCD is essential for genetic recombination and has multiple activities, including dsDNA exonuclease, ssDNA exonuclease, ssDNA endonuclease, DNA-dependent ATPase, and helicase activities (57). It is composed of three subunits: RecB, RecC, and RecD. Thus, as both the RecB and RecD subunits are members of the SF1 helicase superfamily (RecD is a $5^{\prime}-3^{\prime}$ helicase and $\operatorname{RecB}$ is a $3^{\prime}-5^{\prime}$ helicase) (42) RecBCD possesses two ATP-dependent motors (58). Interestingly, the presence of these two different motors allows RecBCD to translocate along ssDNA either from $3^{\prime}$ to $5^{\prime}$ or from $5^{\prime}$ to $3^{\prime}$ (59) (see Figure 3). The rate of RecBCD translocation in the $5^{\prime}-3^{\prime}$ direction along ssDNA is faster than the rate of translocation in the $3^{\prime}-5^{\prime}$ direction (60); at saturating ATP concentrations this rate is $(1515 \pm 33) \mathrm{nt} / \mathrm{s}$ for $3^{\prime}-5^{\prime}$ translocation and $(2037 \pm 109) \mathrm{nt} / \mathrm{s}$ for $5^{\prime}-3^{\prime}$ translocation. There is no difference in the kinetic

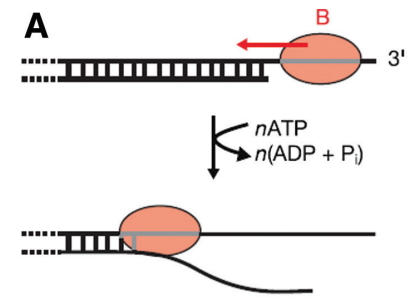

B

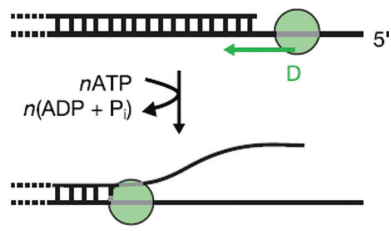

C

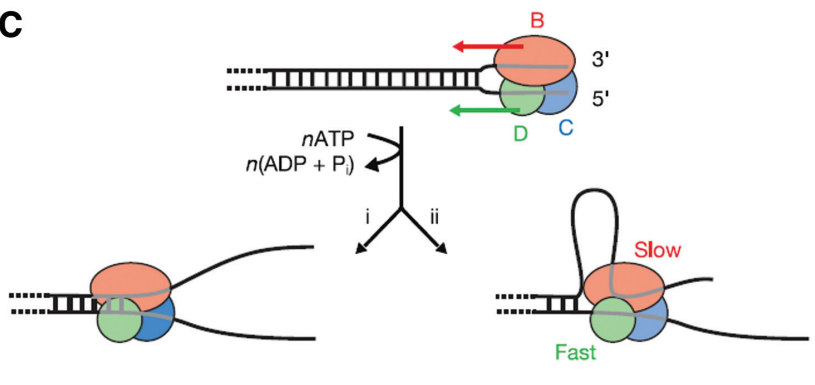

'ssDNA loop-tails' intermediate

Figure $3 \operatorname{RecBCD}$ is shown here as both an example of a multiple motor helicase and a model system for several key translocation concepts.

(A) The RecB motor is a $3^{\prime}-5^{\prime}$ ssDNA translocase. If the motor binds to ssDNA then attempts to translocate into a dsDNA segment it becomes a helicase. (B) The RecD motor has similar behavior to $B$ except it is a $3^{\prime}-5^{\prime}$ ssDNA translocase. (C) If the RecD motor has the same translocation rate as $\operatorname{Rec} B$ then a uniform helicase behavior would occur as shown in the lower left. If the rates differ ssDNA loops can be formed. Image from ref. (58) with permission.

step-size for these two directions of RecBCD translocation and both are $\sim 4$ nucleotides (60).

Isolated monomers of $\operatorname{RecB}$ have been shown to translocate from $3^{\prime}$ to $5^{\prime}$ along ssDNA with a macroscopic rate of $(860 \pm 53) \mathrm{nt} / \mathrm{s}$ and a kinetic step-size of $(4.8 \pm 0.3)$ nucleotides at saturating ATP concentrations (23). Interestingly, the RecBC translocase, which contains only the single RecB motor, is also capable of $5^{\prime}-3^{\prime}$ translocation along ssDNA (23). At saturating ATP concentrations RecBC exhibits $3^{\prime}-5^{\prime}$ translocation with a macroscopic rate and kinetic step-size of $(920 \pm 33) \mathrm{nt} / \mathrm{s}$ and $(3.5 \pm 0.2) \mathrm{nt}$, respectively, and $5^{\prime}-3^{\prime}$ translocation with a macroscopic rate of $(1060 \pm 30) \mathrm{nt} / \mathrm{s}$; when both translocase activities occur simultaneously the macroscopic rate of translocation is $(671 \pm 47) \mathrm{nt} / \mathrm{s}$. Difficulty in determining a kinetic stepsize for $5^{\prime}-3^{\prime}$ translocation by RecBC was attributed to the possible presence of non-uniform processes (pausing, backward motion, etc) that may be occurring during RecBC translocation in this direction. An additional difference between these two translocase activities is that $3^{\prime}-5^{\prime}$ translocation is blocked by reverse polarity linkages in the DNA, but $5^{\prime}-3^{\prime}$ translocation is not (23). Despite 
these differences, ATPase is tightly coupled to translocation for both translocase activities of the RecB motor (29). The coupling efficiency is $(0.81 \pm 0.05) \mathrm{ATP} / \mathrm{nt}$ when the $3^{\prime}-5^{\prime}$ primary translocase is active, $(1.12 \pm 0.06)$ ATP/ nt when the secondary $5^{\prime}-3^{\prime}$ translocase is active, and $(1.07 \pm 0.09) \mathrm{ATP} / \mathrm{nt}$ when both translocase activities are simultaneously active (29).

Monomers of RecD have been shown to translocate from $5^{\prime}$ to $3^{\prime}$ along ssDNA with a macroscopic translocation rate and ATP coupling efficiency of $(95 \pm 5) \mathrm{nt} / \mathrm{s}$ and $(1.03 \pm 0.14) \mathrm{ATP} / \mathrm{nt}$, respectively (61), but a kinetic stepsize for ssDNA translocation has not yet been determined.

SF1 helicases/translocases thus display the following general characteristics in their DNA translocation mechanisms: tight coupling of ATP to translocation, a physical step-size of $\sim 1$ nucleotide, a kinetic step-size greater than one nucleotide, translocation rates on the order of hundreds of nucleotides per second and very high processivity. Furthermore, while DNA translocation by each of these motors appears most consistent with an inchworm model, the difference between the kinetic and physical step-sizes of translocation indicates that these motors likely translocate through a non-uniform process. Furthermore, this non-uniform motion may be associated with the formation and resolution of small (4-5 nucleotide) DNA loops.

\section{SF2 family helicases}

The SF2 family is the largest collection of helicases $(36,43)$. The SF2 helicases whose DNA translocation activity has been most thoroughly studied are RecQ and NS3h. The first member of the RecQ family of helicases was identified in E. coli and has been shown to translocate along ssDNA from $3^{\prime}$ to $5^{\prime}$ with a macroscopic rate and ATP coupling efficiency of $(31 \pm 8) \mathrm{nt} / \mathrm{s}$ and (1.1 \pm 0.2$)$ ATP/nt, respectively (62); similarly, the RecQ family BLM helicase exhibits a coupling efficiency of $1 \mathrm{ATP} / \mathrm{nt}$ during its translocation along ssDNA (63). It has been further estimated that RecQ will translocate between 100 nucleotides and 350 nucleotides on average before dissociation (62). More recent studies have provided estimates of $(16 \pm 4) \mathrm{nt} / \mathrm{s}$ and $(1.6 \pm 0.3) \mathrm{ATP} / \mathrm{nt}$ for the macroscopic translocation rate and ATP coupling efficiency, respectively (64). Interestingly, these same studies also indicate that RecQ displays a non-uniform translocation mechanism in which it moves approximately five nucleotides rapidly before undergoing a slower process that is ratelimiting for the entire reaction (64). This is reminiscent of the translocation mechanisms proposed for the SF1 helicases UvrD and PcrA.
RecQ binds to ssDNA through a two-step mechanism with the second step associated with the binding of the HRDC domain to ssDNA (65); this occurs for all nucleotide bound states of the enzyme (ADP, AMPPNP, and ADP.AlF4 were tested). As the affinity of RecQ for ssDNA binding is highest in the presence of ADP.AlF4 state (65) it has been argued that the rate-limiting process in the ssDNA translocation mechanism for RecQ, possibly associated with the power-stroke of the translocation (see Figure 2), may be linked to either ATP hydrolysis or phosphate release (65). For the BLM helicase the rate-limiting step of the translocation cycle is believed to occur between two ADP-bound enzyme states (63), suggesting that the power-stroke might correspond to ADP release. Interestingly, the ATP hydrolysis activity of RecQ shows little dependence on the DNA sequence but is cooperative with respect to ATP concentration, suggesting that the minimal size of RecQ for ssDNA translocation is at least a dimer (66).

The nonstructural protein 3 helicase (NS3h) of hepatitis $C$ virus is a $3^{\prime}-5^{\prime}$ SF2 RNA and DNA helicase that is essential for the replication of hepatitis $C$ virus (67-70). Monomers of HCV NS3h have been shown to translocate from $3^{\prime}$ to $5^{\prime}$ along ssDNA and ssRNA with a macroscopic rate that depends upon both the base moiety and sugar moiety of the nucleic acid (71). The macroscopic rate at saturating ATP concentrations is $(3.35 \pm 0.09) \mathrm{nt} / \mathrm{s}$ for oligo(dT) translocation, $(35.4 \pm 0.6) \mathrm{nt} / \mathrm{s}$ for oligo(dU) translocation, and (42.2 \pm 1.5$) \mathrm{nt} / \mathrm{s}$ for oligo(rU) translocation; the corresponding kinetic step-sizes are $(1.38 \pm 0.07)$ nucleotides, (1.00 \pm 0.07$)$ nucleotides, and (1.05 \pm 0.08$)$ nucleotides, respectively. Interestingly, despite the differences in macroscopic translocation rates, the ATP coupling efficiencies are identical for all three substrates with NS3h consuming $\sim 0.5$ ATP per nucleotide translocated. This suggests that NS3h also displays non-uniform translocation that is more consistent with a ratchet or inchworm mechanism (34) than with a purely Brownian motor (4).

Interestingly, the affinity of NS3h for binding these translocation substrates is inversely proportional to the associated macroscopic rate of translocation (71), similar to what has been observed for the Rep helicase (51). This suggests that the efficiency with which NS3h can couple ATP binding and hydrolysis to nucleic acid translocation provides a constraint that results in a compensatory relationship between the affinity of nucleic acid binding and the macroscopic rate of nucleic acid translocation (71). Further confirmation of the correlation between substrate binding affinity and DNA translocation is found in a separate study in which a macroscopic rate of $(46 \pm 5) \mathrm{nt} / \mathrm{s}$ for NS3h translocation along oligo(dT) was determined at higher temperature and higher $\mathrm{NaCl}$ concentration (72); an increase 
in both the temperature and the $\mathrm{NaCl}$ concentration would likely decrease the affinity of ssDNA binding by NS3h. This comparison is made slightly problematic, however, by the fact that these two studies used different constructs for NS3h $(71,72)$. Nevertheless, it is worth mentioning that both studies reported similar estimates of the kinetic stepsize of translocation along oligo(dT) [(1.38 \pm 0.07$) \mathrm{nt}(71)$ vs. (1.7 \pm 0.2$) \mathrm{nt}(72)]$ and that an additional slow process occurs during the translocation mechanism $(71,72)$. Candidates for this slow process are an initiation step proceeding processive translocation or a two-step dissociation from the end of the ssDNA $(71,72)$.

It has also been shown recently that the presence of the NS3 protease domain increases the ATP coupling efficiency of NS3h without affecting the macroscopic translocation rate (73). This suggests that interactions between the protease and helicase domains of NS3 improve the energy transduction efficiency of the NS3h motor by improving the allosteric crosstalk between the ATP and nucleic acid binding sites within the NS3h motor or by stabilizing the interaction between the motor and the singlestranded oligonucleotide. The results of this same study also demonstrated that NS3h translocates faster along oligo(rU) than along olidgo(dT) (73), but that the ATP coupling efficiency and kinetic step-size were $5 \mathrm{ATP} / \mathrm{nt}$ and 0.2 nucleotides, respectively (73), again suggesting a nonuniform translocation mechanism for NS3h.

Interestingly, SF2 helicases/translocases are generally slower and less processive DNA translocases than SF1 helicases. This distinction may well be predicated on differences in how nucleotide binding allosterically regulates DNA binding between these two classes of motors. Additional structural and kinetic studies characterizing these allosteric effects are required to explore this hypothesis further. The similarities in the physical step-size between SF1 and SF2 motors (both are $\sim 1$ nucleotide) likely reflects underlying similarities (i.e., common elements) in the ATPase domains of these related classes of proteins (36).

\section{SF3 through SF6 family helicases}

The DNA translocation and unwinding activities of the multimeric helicases comprising the SF3 through SF6 families are naturally regulated by the communication between the individual motors comprising the oligomeric helicase/translocase. Indeed, while the individual motor domains of these complexes share structure/function similarities with the monomeric and dimeric SF1 and SF2 helicases/translocases discussed above, the intracomplex coordination of the motor domains nevertheless dominates the overall function of these enzymes (35, 41, 74). While a thorough discussion of these interactions and processes is beyond the scope of this review, it is nevertheless important to highlight some recent investigations into the DNA translocation activity of these complexes.

The bacteriophage T7 gene product 4 (gp4) is an SF4 hexameric helicase that translocates from $5^{\prime}$ to $3^{\prime}$ along ssDNA $(3,74)$ and is capable of translocating on the order of $75 \mathrm{~kb}$ before dissociation (75). During its translocation along ssDNA T7 gp4 moves two to three nucleotides for each molecule of ATP it hydrolyzes (75). The results of recent single-molecule studies indicate that T7 gp4 translocates along ssDNA with a macroscopic rate of $(409 \pm 17) \mathrm{nt} / \mathrm{s}(76)$ or $\sim 600 \mathrm{nt} / \mathrm{s}(77)$. This rate was further shown to depend upon the base moiety of the nucleotide hydrolyzed, with fastest translocation for adenosine and slowest for guanine (77).

The hexameric SF4 helicase DnaB from $E$. coli unwinds dsDNA ahead of the replication fork thereby providing ssDNA templates for the DNA polymerase II holoenzyme (78). The results of recent single-molecule experiments indicate a macroscopic rate of $(390 \pm 15) \mathrm{nt} / \mathrm{s}$ for the translocation of DnaB along ssDNA (79).

The large $\mathrm{T}$ antigen helicase from Simian Virus 40 is an SF6 helicase that has been shown to translocate from $3^{\prime}$ to $5^{\prime}$ along ssDNA (80), but neither a macroscopic rate nor ATP coupling efficiency have been determined.

\section{DNA translocation by chromatin remodeling enzymes}

Most eukaryotic DNA is packaged into nucleosomes that render these sequences largely inaccessible for transcription or repair $(81,82)$. Molecular motors called chromatin remodelers can relieve the inhibition of these processes by sliding or disassembling the nucleosomes (7-9) and thereby serve an essential role in the regulation of gene expression (83). Based upon their highly conserved ATPase domain chromatin remodelers are classified as part of a large Snf2 family of proteins, which in turn is part of the helicase superfamily SF2 (Figure 4) $(84,85)$. Chromatin remodelers lack helicase activity (86), but they share the ATP-dependent DNA translocation activity of helicases (27, 87-90). Furthermore, nucleosome repositioning activity is underpinned by DNA translocation in most models (85, 91-95). Qualitative demonstrations of DNA translocation by chromatin remodelers, have recently been extended by quantitative determinations of the kinetic mechanism of translocation $(87,88,96,97)$. 


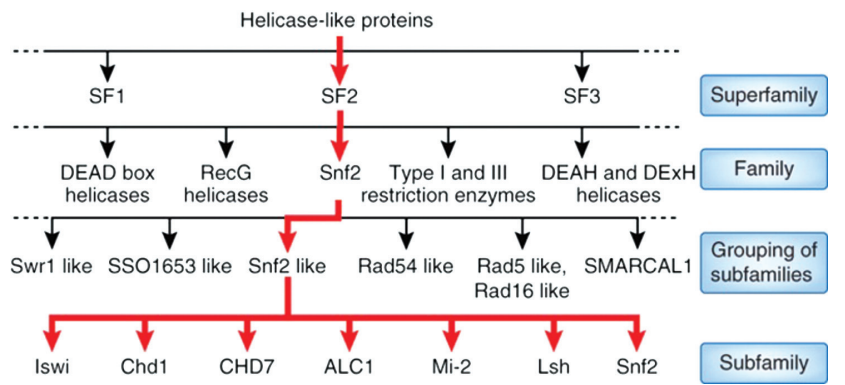

Figure 4 This hierarchical grouping of helicase-like proteins shows how even an abbreviated list of the common helicase-like proteins hints at the highly diverse behaviors found within the translocase umbrella. Image taken from ref. (85) with permission.

The RSC chromatin remodeling complex is a member of the SWI/SNF family of chromatin remodelers and is capable of repositioning nucleosomes from the center of DNA fragments toward the ends without disrupting the integrity of the nucleosomes $(98,99)$. Recently both ensemble and single-molecule measurements have independently demonstrated that the motor subunit of RSC is a poorly processive dsDNA translocase $(27,32,100)$, as it moves fewer than 40 basepairs, on average before dissociating from the DNA. The macroscopic translocation rate and kinetic step-size for RSC motor translocation were determined in ensemble studies to be $(2.9 \pm 0.1) \mathrm{bp} / \mathrm{s}$ and (1.24 \pm 0.18$)$ basepairs, respectively (27). Combined with the results of these same studies indicating a coupling efficiency of $(3.0 \pm 0.4) \mathrm{ATP} / \mathrm{bp}$, a physical step-size of approximately three basepairs is indicated. The disparity between the kinetic and physical step-sizes indicates that RSC likely translocates along dsDNA according to a non-uniform stepping model (27). However, unlike the previously discussed non-uniform mechanisms for UvrD and Rep, for RSC the physical step size is larger than the kinetic step-size.

Indeed, a physical step-size larger than a kinetic stepsize suggests that futile ATP hydrolysis occurs during dsDNA translocation by RSC. Furthermore, this futile hydrolysis might simply result from the fact that RSC is more prone to dissociate from the DNA during translocation than SF1 helicases such as UvrD and Rep. While this might be a trivial consequence of differences in the allosteric regulation of DNA binding by ATP binding for these motors, it may indicate a difference in the timescales associated with the physical processes associated with translocation. Indicating RSC may spend a larger fraction of its translocation cycle in a state with lower DNA binding affinity than other motors, such as UvrD and Rep. Consistent with this hypothesis is the observation that the macroscopic rate of DNA translocation by RSC is at least an order of magnitude smaller than that of UvrD or Rep.

RSC's core translocation motor has been utilized in single-molecule experiments combining the tethering of the motor to the DNA and optical tweezers to measure the forces involved in translocation. From this a macroscopic rate of $\sim 25 \mathrm{bp} / \mathrm{s}$ and kinetic step-size of $\sim 2$ basepairs were measured (32). Interestingly, these results also indicated that during translocation the RSC motor typically forms dsDNA loops of a few basepairs. This provides further evidence for a non-uniform translocation mechanism for the RSC motor (27). Additionally, the single-molecule experiments uncovered a tendency to occasionally form loops of $\sim 30$ basepairs (32). This result provides support for the model that DNA loops are a fundamental intermediate in the nucleosome repositioning activity of RSC $(32,87,90$, 101). Furthermore, the affinity of dsDNA binding by the RSC motor is reduced in the presence of both ADP and ATP analogs (102). The reduction in affinity during the ATPase portion of the translocation cycle, may account for the poor processivity of dsDNA translocation by the RSC motor (27, 32). RSC's poor processivity may underlay its physical step-size for dsDNA translocation being larger than the corresponding kinetic step-size.

When the RSC core motor is in its full complex it exhibits a macroscopic translocation rate of $\sim 13 \mathrm{bp} / \mathrm{s}$ and is capable of creating dsDNA loops with an average size of $\sim 100$ basepairs (90). Interestingly, a slow mandatory initiation process following dsDNA binding is observed before DNA translocation begins $(27,100)$; it should be noted that it is still unclear if this process was ATP-dependent or represented an ATP-independent conformational change following DNA binding by RSC. A multi-step DNA binding mechanism may be a common theme in all translocases, as it has also been found in the ISW2 chromatin remodeler (103), the human polymerase $\beta(104,105)$, and several helicases $(24,75,106-108)$.

While the mechanisms of DNA translocation of few chromatin remodelers have been determined, it is nevertheless important to note the similarities to other SF2 family translocases. Specifically, these motors all exhibit show rates of translocation (tens of basepairs or nucleotides per second) and very poor processivity.

\section{DNA translocation by restriction enzymes}

Type I DNA restriction/modification enzymes are members of the Snf2 family of SF2 helicases (see Figure 4) 
and cleave specific unmethylated DNA sequences (12, 13). The cleavage reaction begins with the recognition of the unmethylated target sequence by the complex's methyltransferase core enzyme $(12,13)$. Initial binding is subsequently followed by ATP-dependent dsDNA translocation, performed by the two HsdR complex subunits, which pulls DNA toward the core enzyme and forming two dsDNA loops $(109,110)$. The translocating subunits then conduct the cleavage reaction upon encountering a blockage on the DNA, including encountering a second restriction enzyme (111).

The type I DNA restriction/modification enzyme EcoR124I is capable of translocating along dsDNA with an average macroscopic rate of $(550 \pm 30) \mathrm{bp} / \mathrm{s}$ (110). EcoR124I is also highly processive as it is capable of translocating an average of $(4300 \pm 900)$ basepairs before dissociation (110). During its processive translocation along dsDNA EcoR124I consumes $\sim 1$ ATP per basepair, indicating that the physical step-size of translocation is $\sim 1$ basepair, and moves with a kinetic step-size of $1.9 \pm 0.6$ basepairs (112). Both the tight coupling of ATP hydrolysis to translocation and the high processivity of translocation suggest an inchworm mechanism for DNA translocation by EcoR124I. The small difference between the physical and kinetic stepsizes may indicate that EcoR124I also experiences nonuniform translocation.

\section{DNA translocation by viral packaging motors}

Bacteriophages and herpesviruses encode a DNA translocase that is responsible for packaging the viral genome into the preformed capshid shell or prohead (10). These translocases, called terminases, are capable of ATP-dependent dsDNA translocation against high force (10) in order to compact the genome to near-crystalline density (11).

Bacteriophage lambda is a dsDNA virus that infects E. coli (113). Lambda utilizes rolling circle DNA replication resulting in concatemers (i.e., long linear DNA). Lambda terminase is then responsible for packing the concatemer into the viral capsid (113). During the packaging reaction lambda terminase translocates along the double-stranded viral DNA in an ATP-dependent reaction (114) with a macroscopic rate and kinetic step-size of $(119 \pm 8) \mathrm{bp} / \mathrm{s}$ and (410 \pm 150$)$ basepairs at $4^{\circ} \mathrm{C}$ and saturating ATP concentration (115). It is further argued that the kinetic step-size is not a physical step-size, but rather corresponds to periodic pausing by lambda terminase during the packaging reaction (115). In other words, lambda terminase also experiences non-uniform translocation. Indeed, pausing in the packaging reaction were also detected in singlemolecule based experiments (33), which also reported a macroscopic translocation rate of $\sim 600 \mathrm{bp} / \mathrm{s}$ at $23^{\circ} \mathrm{C}$ (115). The macroscopic translocation rate was shown to decrease throughout the packaging reaction as increasing resistive force was built up by the packaged DNA within the interior of the capsid (33).

The macroscopic rate of DNA translocation by the Bacillus subtilis phage $\phi 29$ packaging motor also decreases during the course of the packaging reaction (116). However, the maximum rate observed at the start of the reaction, $\sim 100 \mathrm{bp} / \mathrm{s}$ (116), is much slower than the rate observed for bacteriophage lambda terminase (115) under similar conditions. Similar to what is observed with bacteriophage lambda terminase, frequent pausing is observed during the packaging of the phage $\phi 29$ genome (116) suggesting a related mechanism of packaging by both motors. The rate of packaging by the phage $\phi 29$ packaging motor has also been shown to be significantly affected by ionic screening, indicating the importance of electrostatic repulsing during the packaging reaction (117), and furthermore an ATP coupling efficiency of 0.5 ATP/bp has been determined for this motor (118).

\section{DNA translocation by other motors}

The Polo-like kinase 1 (Plk1) is a key regulator of mitosis, meiosis and cytokinesis in vertebrates (119). The Plk1interacting checkpoint helicase (PICH) is an Snf2 family SF2 helicase (see Figure 4) that controls the localization of Plk1 and serves as an essential component of mitotic checkpoint signaling (120). The macroscopic rate of translocation of PICH along dsDNA is dependent upon the concentration of $\mathrm{NaCl}$ and varies from $\sim 22 \mathrm{bp} / \mathrm{s}$ at low $\mathrm{NaCl}$ concentration to $\sim 30 \mathrm{bp} / \mathrm{s}$ at high $\mathrm{NaCl}$ concentration (121). As the affinity of PICH for binding DNA decreases as the concentration of $\mathrm{NaCl}$ in solution increases (121), it is possible that, similar to what is observed with the SF2 helicase NS3h (71), an allosteric and compensatory relationship between the affinity of nucleic acid binding and the macroscopic rate of nucleic acid translocation exists for PICH.

The ability of $E$. coli FtsK to translocate along dsDNA enables the enzyme to pump chromosomal DNA through the closing septum during cell division (122). FtsK is not classified as a member of any helicase family, but is a member of the large AAA+ superfamily (123) of proteins, which also includes helicases; FtsK is specifically 
a member of the SpoIIIE/FtsK family of AAA+ proteins, which includes many membrane-anchored DNA translocases responsible for chromosome separation in bacteria $(123,124)$. FtsK is a remarkable dsDNA translocase in that it exhibits macroscopic translocation rates on the order of 5-8 kb/s (125-127) and corresponding very high processivities. Although neither a kinetic step-size nor ATP coupling efficiency for dsDNA translocation by FtsK has been determined, several DNA sequences that control the direction of FtsK translocation have been identified $(128,129)$.

\section{Conclusion}

We see the trend of new results continuing to confirm that conserved sequence motifs, traditionally described as helicase motifs, should more accurately be referred to as DNA helicase/translocase motifs as they are found across a wide variety of DNA translocases, including those which display no helicase activity. This trend has been previously discussed (35), but we would like to reinforce that the evidence is growing and the general thinking of the topic should shift to reflect the evidence. Within this context it is import to recognize that many translocases within the larger 'helicase' family of motor proteins share a variety of common structure/function relationships and mechanistic themes that bear further elucidation. An important example, is the relation between members of SF1 and SF2 as both have been observed to translocate along DNA with coupling efficiencies $\sim 1$ or $2 \mathrm{ATP} / \mathrm{nt}$ or ATP/bp, but SF1 translocases generally have much higher macroscopic translocation rates and are much more processive than SF2 translocases. The similar coupling efficiencies are, perhaps, to be expected given the common structural elements possessed by these different motors (indeed, this is the basis for their common classification); however the origin of the differences in rate and processivity is not immediately clear. One possibility is that the difference in the effect of ATP/ADP allosterically regulated binding to DNA provides an underlying behavioral shift between members of SF1 and SF2 that accounts for some of the disparities in rate and processivity between members of these groups. More complete and comprehensive studies of the mechanisms of DNA binding by these motors are required to test such a hypothesis. Similarly, additional structural and computational modeling work is necessary to determine if there is an inherent mechanical difference in the physical interactions of these motors with DNA that accounts for the variance in their translocation abilities. For example, perhaps the SF2 translocases simply spend more time during their translocation cycle in states with lower DNA affinity than SF1 helicases.

Such systematic studies of DNA binding, especially when performed under a variety of solution conditions and coupled with associated determinations of the macroscopic rate and ATP coupling efficiency of DNA translocation, would enable us to test the universality of the apparent inverse relationship between translocation rate and DNA binding affinity. As all helicases/translocases use a similar domain as their mechanism for energy transduction during translocation it is not surprising that all of these motors might share a common fundamental physical step-size and ATP coupling efficiency for tanslocation. It is then possible that this mechanism also provides a mechanical constraint that creates the inverse proportionality between rate and affinity.

Another common, and perhaps universal, theme of DNA translocation shared by SF1 and SF2 motors is that they translocate along DNA with a non-uniform mechanism that is likely associated with the formation of DNA loops. While it is not known if the formation of ssDNA loops by SF1 helicases during their translocation has any implications for their activity in vivo, dsDNA loop formation by chromatin remodelers and restriction enzymes is an integral part of their function. It is tempting to speculate on the implications of these observations for understanding the evolutionary origin of these motors. Loop formation may well be a simple and potentially initially innocuous consequence of the structural changes occurring within the motor as it translocates along the DNA. Eventually this inherent result of DNA translocation might have then been integrated into a variety of cellular activities.

Similarly, a common theme in the determined mechanisms of DNA translocation is the occurrence of additional slow processes, possibly associated with the initiation of translocation or a multi-step dissociation from the end of the DNA. It is worth noting that a two-step mechanism of DNA binding is also common among these motors, although few complete characterizations of DNA binding by translocases have been completed. Perhaps the results of these two sets of experiments (translocation and binding) are sensitive to the same mechanical process? A thorough combined determination of the mechanisms of DNA binding and translocation by these motors is required to test the validity of this speculation. Of course, such binding studies should also be organized to identify the allosteric regulation of the mechanisms of DNA binding by ATP/ADP binding as this would complete the equilibrium DNA binding experiments discussed above. 


\section{References}

1. Lohman TM, Hsieh J, Maluf NK, Cheng W, Lucius AL, Fischer CJ, Brendza KM, Korolev S, Waksman G. DNA helicases, motors that move along nucleic acids: lessons from the SF1 helicase superfamily. In: Hackney DD, Tamanoi F, editors. The enzymes. London, UK: Elsevier Academic Press, 2003.

2. Pyle AM. Translocation and unwinding mechanisms of RNA and DNA helicases. Annu Rev Biophys 2008; 37: 317-36.

3. Patel SS, Picha KM. Structure and function of hexameric helicases. Annu Rev Biochem 2000; 69: 651-97.

4. Patel SS, Donmez I. Mechanisms of helicases. J Biol Chem 2006; 281: $18265-8$.

5. Lohman TM, Tomko EJ, Wu CG. Non-hexameric DNA helicases and translocases: mechanisms and regulation. Nat Rev Mol Cell Biol 2008; 9: 391-401.

6. Matson SW, Kaiser-Rogers KA. DNA helicases. Annu Rev Biochem 1990; 59: 289-329.

7. Becker PB, Horz W. ATP-dependent nucleosome remodeling. Annu Rev Biochem 2002; 71: 247-73.

8. Clapier CR, Cairns BR. The biology of chromatin remodeling complexes. Annu Rev Biochem 2009; 78: 273-304.

9. Saha A, Wittmeyer J, Cairns BR. Chromatin remodelling: the industrial revolution of DNA around histones. Nat Rev Mol Cell Biol 2006; 7: 437-47.

10. Feiss M, Rao VB. The bacteriophage DNA packaging machine. Adv Exp Med Biol 2012; 726: 489-509.

11. Earnshaw WC, Casjens SR. DNA packaging by the doublestranded DNA bacteriophages. Cell 1980; 21: 319-31.

12. Yuan R. Structure and mechanism of multifunctional restriction endonucleases. Annu Rev Biochem 1981; 50: 285-319.

13. Bickle TA, Krüger DH. Biology of DNA restriction. Microbiol Rev 1993; 57: 434-50.

14. Stanley LK, Seidel R, van der Scheer C, Dekker NH, Szczelkun $M D$, Dekker C. When a helicase is not a helicase: dsDNA tracking by the motor protein EcoR124I. EMBO J 2006; 25: 2230-9.

15. Meisel A, Mackeldanz P, Bickle TA, Krüger DH, Schroeder C. Type III restriction endonucleases translocate DNA in a reaction driven by recognition site-specific ATP hydrolysis. EMBO J 1995; 14: 2958-66.

16. Crampton N, Roes S, Dryden DT, Rao DN, Edwardson JM, Henderson RM. DNA looping and translocation provide an optimal cleavage mechanism for the type III restriction enzymes. EMBO J 2007; 26: 3815-25.

17. Crampton N, Yokokawa M, Dryden DT, Edwardson JM, Rao DN, Takeyasu K, Yoshimura SH, Henderson, RM. Fast-scan atomic force microscopy reveals that the type III restriction enzyme EcoP15I is capable of DNA translocation and looping. Proc Natl Acad Sci USA 2007; 104: 12755-60.

18. Smith RM, Josephsen J, Szczelkun MD. The single polypeptide restriction-modification enzyme LlaGl is a self-contained molecular motor that translocates DNA loops. Nucleic Acids Res 2009; 37: 7219-30.

19. Lucius AL, Maluf NK, Fischer CJ, Lohman TM. General methods for analysis of sequential "n-step" kinetic mechanisms: application to single turnover kinetics of helicase-catalyzed DNA unwinding. Biophys J 2003; 85: 2224-39.
20. Tomko EJ, Fischer CJ, Lohman TM. Ensemble methods for monitoring enzyme translocation along single stranded nucleic acids. Methods 2010; 51: 269-76.

21. Fischer CJ, Wooten L, Tomko EJ, Lohman, TM. Kinetics of motor protein translocation on single-stranded DNA. Methods Mol Biol 2010; 587: 45-56.

22. Tomko EJ, Fischer CJ, Lohman, TM. Single-stranded DNA translocation of E. coli UvrD monomer is tightly coupled to ATP hydrolysis. J Mol Biol 2012; 418: 32-46.

23. Wu CG, Bradford C, Lohman TM. Escherichia coli RecBC helicase has two translocase activities controlled by a single ATPase motor. Nat Struct Mol Biol 2010; 17: 1210-7.

24. Dillingham MS, Wigley DB, Webb MR. Direct measurement of single-stranded DNA translocation by PcrA helicase using the fluorescent base analogue 2-aminopurine. Biochemistry 2002; 41: 643-51.

25. Ordonez H, Shuman S. Mycobacterium smegmatis Lhr Is a DNAdependent ATPase and a $3^{\prime}$-to-5' DNA translocase and helicase that prefers to unwind $3^{\prime}$-tailed RNA:DNA hybrids. J Biol Chem 2013; 288: 14125-34.

26. Yakovleva L, Shuman S. Mycobacterium smegmatis SftH exemplifies a distinctive clade of superfamily II DNA-dependent ATPases with $3^{\prime}$ to $5^{\prime}$ translocase and helicase activities. Nucleic Acids Res 2012; 40: 7465-75.

27. Eastlund A, Malik S, Fischer CJ. Determination of the kinetic mechanism of double-stranded DNA translocation by S. cerevisiae RSC. Arch Biochem Biophys 2013; 532: 73-83.

28. Tomko EJ, Fischer CJ, Niedziela-Majka A, Lohma TM. A nonuniform stepping mechanism for E. coli UvrD monomer translocation along single-stranded DNA. Mol Cell 2007; 26: 335-47.

29. Wu CG, Xie F, Lohman TM. The primary and secondary translocase activities within E. coli RecBC helicase are tightly coupled to ATP hydrolysis by the RecB motor. J Mol Biol 2012; 423: 303-14.

30. Dillingham MS, Wigley DB, Webb, MR. Demonstration of unidirectional single-stranded DNA translocation by PcrA helicase: measurement of step size and translocation speed. Biochemistry 2000; 39: 205-12.

31. Ramanagoudr-Bhojappa R, Chib S, Byrd AK, Aarattuthodiyil S, Pandey M, Patel SS, Raney KD. Yeast Pif1 helicase exhibits a one-base-pair stepping mechanism for unwinding duplex DNA. J Biol Chem 2013; 288: 16185-95.

32. Sirinakis G, Clapier CR, Gao Y, Viswanathan R, Cairns BR, Zhang Y. The RSC chromatin remodelling ATPase translocates DNA with high force and small step size. EMBO J 2011; 30: 2364-72.

33. Fuller DN, Raymer DM, Rickgauer JP, Robertson RM, Catalano CE, Anderson DL, Grimes S, Smith DE. Measurements of single DNA molecule packaging dynamics in bacteriophage lambda reveal high forces, high motor processivity, and capsid transformations. J Mol Biol 2007; 373: 1113-22.

34. Gu M, Rice CM. Three conformational snapshots of the hepatitis $C$ virus NS3 helicase reveal a ratchet translocation mechanism. Proc Natl Acad Sci USA 2010; 107: 521-8.

35. Enemark EJ, Joshua-Tor L. On helicases and other motor proteins. Curr Opin Struct Biol 2008; 18: 243-57.

36. Fairman-Williams ME, Guenther U-P, Jankowsky E. SF1 and SF2 helicases: family matters. Curr Opin Struct Biol 2010; 20: 313-24.

37. Levin MK, Gurjar M, Patel SS. A Brownian motor mechanism of translocation and strand separation by hepatitis $C$ virus helicase. Nat Struct Mol Biol 2005; 12: 429-35. 
38. Zheng W, Tekpinar M. Structure-based simulations of the translocation mechanism of the hepatitis $C$ virus NS3 helicase along single-stranded nucleic acid. Biophys J 2012; 103: 1343-53.

39. Lohman, TM. Mechanisms of helicase-catalyzed DNA unwinding. Annu Rev Biochem 1996; 65: 169-214.

40. Matson SW, Bean DW, George JW. DNA helicases: enzymes with essential roles in all aspects of DNA metabolism. Bioessays 1994; 16: 13-22.

41. Singleton MR, Dillingham MS, Wigley DB. Structure and mechanism of helicases and nucleic acid translocases. Annu Rev Biochem 2007; 76: 23-50.

42. Gorbalenya AE, Koonin EV. Helicases: amino-acid sequence comparisons and structure-function relationships. Curr Opin Struct Biol 1993; 3: 419-29.

43. Umate P, Tuteja N, Tuteja R. Genome-wide comprehensive analysis of human helicases. Commun Integr Biol 2011; 4: 118-37.

44. Hall MC, Jordan JR, Matson SW. Evidence for a physical interaction between the Escherichia coli methyl-directed mismatch repair proteins MutL and UvrD. EMBO J 1998; 17: 1535-41.

45. Fischer CJ, Maluf NK, Lohman TM. Mechanism of ATP-dependent translocation of E.coli UvrD monomers along single-stranded DNA. J Mol Biol 2004; 344: 1287-309.

46. Lee KS, Balci H, Jia H, Lohman TM, Ha T. Direct imaging of single UvrD helicase dynamics on long single-stranded DNA. Nat Commun 2013; 4: 1878.

47. Lu HP, Xun L, Xie XS. Single-molecule enzymatic dynamics. Science $1998 ; 282: 1877-82$.

48. Xie XS. Single-molecule approach to dispersed kinetics and dynamic disorder: probing conformational fluctuation and enzymatic dynamics. J Chem Phys 2002; 117: 11024-32.

49. Khan SA. Plasmid rolling-circle replication: recent developments. Mol Microbiol 2000; 37: 477-84.

50. Chang T-L, Naqvi A, Anand SP, Kramer MG, Munshi R, Khan SA. Biochemical characterization of the Staphylococcus aureus PcrA helicase and its role in plasmid rolling circle replication. J Biol Chem 2002; 277: 45880-6.

51. Brendza KM, Cheng W, Fischer CJ, Chesnik MA, Niedziela-Majka A, Lohman TM. Autoinhibition of Escherichia coli Rep monomer helicase activity by its 2B subdomain. Proc Natl Acad Sci USA 2005; 102: 10076-81.

52. Niedziela-Majka A, Chesnik MA, Tomko EJ, Lohman, TM. Bacillus stearothermophilus PcrA monomer is a single-stranded DNA translocase but not a processive helicase in vitro. J Biol Chem 2007; 282: 27076-85.

53. Chisty LT, Toseland CP, Fili N, Mashanov GI, Dillingham MS, Molloy JE, Webb MR Monomeric PcrA helicase processively unwinds plasmid lengths of DNA in the presence of the initiator protein RepD. Nucleic Acids Res 2013; 41: 5010-23.

54. Toseland CP, Martinez-Senac MM, Slatter AF, Webb MR. The ATPase Cycle of PcrA helicase and its coupling to translocation on DNA. J Mol Biol 2009; 392: 1020-32.

55. Marians KJ. Mechanisms of replication fork restart in Escherichia coli. Philos Trans R Soc Lond B Biol Sci 2004; 359: 71-7.

56. Denhardt DT, Dressler DH, Hathaway A. The abortive replication of PhiX174 DNA in a recombination-deficient mutant of Escherichia coli. Proc Natl Acad Sci USA 1967; 57: 813-20.

57. Dillingham MS, Kowalczykowski, SC. RecBCD enzyme and the repair of double-stranded DNA breaks. Microbiol Mol Biol Rev 2008; 72: 642-71.

58. Dillingham MS, Spies M, Kowalczykowski SC. RecBCD enzyme is a bipolar DNA helicase. Nature 2003; 423: 893-7.
59. Chung C, Li H-W. Direct observation of RecBCD helicase as singlestranded DNA translocases. J Am Chem Soc 2013; 135: 8920-5.

60. Xie F, Wu CG, Weiland E, Lohman, TM. Asymmetric regulation of bipolar single-stranded DNA translocation by the two motors within Escherichia coli RecBCD helicase. J Biol Chem 2013; 288: 1055-64.

61. Saikrishnan K, Powell B, Cook NJ, Webb MR, Wigley DB. Mechanistic basis of $5^{\prime}-3^{\prime}$ translocation in SF1B helicases. Cell 2009; 137: 849-9.

62. Sarlos K, Gyimesi M, Kovacs M. RecQ helicase translocates along single-stranded DNA with a moderate processivity and tight mechanochemical coupling. Proc Natl Acad Sci USA 2012; 109: 9804-9.

63. Gyimesi M, Sarlós K, Kovács M. Processive translocation mechanism of the human Bloom's syndrome helicase along singlestranded DNA. Nucleic Acids Res 2010; 38: 4404-14.

64. Rad B, Kowalczykowski SC. Efficient coupling of ATP hydrolysis to translocation by RecQ helicase. Proc Natl Acad Sci USA 2012; 109: 1443-8.

65. Kocsis ZS, Sarlos K, Harami GM, Martina M, Kovacs M. A Nucleotide- and HRDC-domain-dependent structural transition in DNA-bound RecQ helicase. J Biol Chem 2014; 289: 5938-49.

66. Rad B, Kowalczykowski SC. Translocation of E. coli RecQ helicase on single-stranded DNA. Biochemistry 2012; 51: 2921-9.

67. Bartenschlager R, Lohmann V. Replication of hepatitis C virus. J Gen Virol 2000; 81: 1631-48.

68. Kim DW, Gwack Y, Han JH, Choe J. C-terminal domain of the hepatitis C virus NS3 protein contains an RNA helicase activity. Biochem Biophys Res Commun 1995; 215: 160-6.

69. Suzich JA, Tamura JK, Palmer-Hill F, Warrener P, Grakoui A, Rice CM, Feinstone SM, Collett MS. Hepatitis C virus NS3 protein polynucleotide-stimulated nucleoside triphosphatase and comparison with the related pestivirus and flavivirus enzymes. J Virol 1993; 67: 6152-8.

70. Kolykhalov AA, Mihalik K, Feinstone SM, Rice CM. Hepatitis C virus-encoded enzymatic activities and conserved RNA elements in the $3^{\prime}$ nontranslated region are essential for virus replication in vivo. J Virol 2000; 74: 2046-51.

71. Khaki AR, Field C, Malik S, Niedziela-Majka A, Leavitt SA, Wang R, Hung M, Sakowicz R, Brendza KM, Fischer CJ. The macroscopic rate of nucleic acid translocation by hepatitis $C$ virus helicase NS3h is dependent on both sugar and base moieties. J Mol Biol 2010; 400: 354-78.

72. Matlock DL, Yeruva L, Byrd AK, Mackintosh SG, Langston C, Brown C, Cameron CE, Fischer CJ, Raney KD. Investigation of translocation, DNA unwinding, and protein displacement by NS3h, the helicase domain from the hepatitis C virus helicase. Biochemistry 2010; 49: 2097-109.

73. Rajagopal V, Gurjar M, Levin MK, Patel SS. The protease domain increases the translocation stepping efficiency of the hepatitis $C$ virus NS3-4A helicase. J Biol Chem 2010; 285: 17821-32.

74. Donmez I, Patel SS. Mechanisms of a ring shaped helicase. Nucleic Acids Res 2006; 34: 4216-24.

75. Kim, D-E, Narayan M, Patel SS. T7 DNA helicase: a molecular motor that processively and unidirectionally translocates along single-stranded DNA. J Mol Biol 2002; 321: 807-19.

76. Ribeck N, Saleh OA. DNA unwinding by ring-shaped T4 helicase gp41 is hindered by tension on the occluded strand. PLoS One 2013; 8: e79237.

77. Sun B, Johnson DS, Patel G, Smith BY, Pandey M, Patel SS, Wang MD. ATP-induced helicase slippage reveals highly coordinated subunits. Nature 2011; 478: 132-5. 
78. Schaeffer PM, Headlam MJ, Dixon NE. Protein-protein interactions in the eubacterial replisome. IUBMB Life 2005; 57: 5-12.

79. Ribeck N, Kaplan DL, Bruck I, Saleh OA. DnaB helicase activity is modulated by DNA geometry and force. Biophys J 2010; 99: 2170-9.

80. Yardimci H, Wang X, Loveland AB, Tappin I, Rudner DZ, Hurwitz J, van Oijen AM, Walter JC. Bypass of a protein barrier by a replicative DNA helicase. Nature 2012; 492: 205-9.

81. Ehrenhofer-Murray AE. Chromatin dynamics at DNA replication, transcription and repair. Eur J Biochem 2004; 1: 2335-49.

82. Elgin SC, Grewal SI. Heterochromatin: silence is golden. Curr Biol 2003; 13: R895-8.

83. Wu C. Chromatin remodeling and the control of gene expression. J Biol Chem 1997; 272: 28171-4.

84. Eisen JA, Sweder KS, Hanawalt PC. Evolution of the SNF2 family of proteins: subfamilies with distinct sequences and functions. Nucleic Acids Res 1995; 23; 2715-23.

85. Mueller-Planitz F, Klinker H, Becker PB. Nucleosome sliding mechanisms: new twists in a looped history. Nat Struct Mol Biol 2013; 20: 1026-32.

86. Cote J, Peterson CL, Workman JL. Perturbation of nucleosome core structure by the SWI/SNF complex persists after its detachment, enhancing subsequent transcription factor binding. Proc Natl Acad Sci USA 1998; 95: 4947-52.

87. Saha A, Wittmeyer J, Cairns BR. Chromatin remodeling by RSC involves ATP-dependent DNA translocation. Genes Dev 2002; 16: $2120-34$.

88. Whitehouse I, Stockdale C, Flaus A, Szczelkun MD, Owen-Hughes T. Evidence for DNA translocation by the ISWI chromatin-remodeling enzyme. Mol Cell Biol 2003; 23: 1935-45.

89. Lia G, Indrieri M, Owen-Hughes T, Finzi L, Podesta A, Milani P, Dunlap D. ATP-dependent looping of DNA by ISWI. J Biophotonics 2008; 1: 280-6.

90. Zhang Y, Smith CL, Saha A, Grill SW, Mihardja S, Smith SB, Cairns BR, Peterson CL, Bustamante C. DNA translocation and loop formation mechanism of chromatin remodeling by SWI/SNF and RSC. Mol Cell 2006; 24: 559-68.

91. Zofall M, Persinger J, Kassabov SR, Bartholomew B. Chromatin remodeling by ISW2 and SWI/SNF requires DNA translocation inside the nucleosome. Nat Struct Mol Biol 2006; 13: 339-46.

92. Saha A, Wittmeyer J, Cairns BR. Chromatin remodeling through directional DNA translocation from an internal nucleosomal site. Nat Struct Mol Biol 2005; 12: 747-55.

93. Mueller-Planitz F, Klinker H, Ludwigsen J, Becker PB. The ATPase domain of ISWI is an autonomous nucleosome remodeling machine. Nat Struct Mol Biol 2012; 20: 82-9.

94. Strohner R, Wachsmuth M, Dachauer K, Mazurkiewicz J, Hochstatter J, Rippe K, Langst G. A “loop recapture” mechanism for ACF-dependent nucleosome remodeling. Nat Struct Mol Biol 2005; 12: 683-90.

95. Eberharter A, Vetter I, Ferreira R, Becker PB. ACF1 improves the effectiveness of nucleosome mobilization by ISWI through PHDhistone contacts. EMBO I 2004; 23:4029-39.

96. Lia G, Praly E, Ferreira H, Stockdale C, Tse-Dinh YC, Dunlap D, Croquette V, Bensimon D, Owen-Hughes T. Direct observation of DNA distortion by the RSC complex. Mol Cell 2006; 21: 417-25.

97. Deakyne JS, Huang F, Negri J, Tolliday N, Cocklin S, Mazin AV. Analysis of the activities of RAD54, a SWI2/SNF2 protein, using a specific small-molecule inhibitor. J Biol Chem 2013; 288: 31567-80.
98. Cairns BR, Lorch Y, Li Y, Zhang M, Lacomis L, ErdjumentBromage H, Tempst P, Du J, Laurent B, Kornberg RD. RSC, an essential, abundant chromatin-remodeling complex. Cell 1996; 87: $1249-60$.

99. Lorch Y, Zhang M, Kornberg RD. RSC unravels the nucleosome. Mol Cell 2001; 7: 89-95.

100. Fischer CJ, Saha A, Cairns BR. Kinetic model for the ATPdependent translocation of Saccharomyces cerevisiae RSC along double-stranded DNA. Biochemistry 2007; 46: 12416-26.

101. Wittmeyer J, Saha A, Cairns B. DNA translocation and nucleosome remodeling assays by the RSC chromatin remodeling complex. Methods Enzym 2004; 377: 322-43.

102. Malik SS, Rich E, Viswanathan R, Cairns BR, Fischer CJ. Allosteric interactions of DNA and nucleotides with S. cerevisiae RSC. Biochemistry 2011; 50: 7881-90.

103. Fischer CJ, Yamada K, Fitzgerald DJ. Kinetic Mechanism for Single stranded DNA binding and Translocation by S. cerevisiae Isw2. Biochemistry 2009; 48: 2960-8.

104. Rajendran S, Jezewska MJ, Bujalowski W. Multiple-step kinetic mechanisms of the ssDNA recognition process by human polymerase beta in its different ssDNA binding modes. Biochemistry 2001; 40: 11794-810.

105. Rajendran S, Jezewska MJ, Bujalowski W. Recognition of template-primer and gapped DNA substrates by the human DNA polymerase $\beta$. J Mol Biol 2001; 308: 477-500.

106. Maluf NK, Fischer CJ, Lohman TM. A Dimer of Escherichia coli UvrD is the active form of the helicase in vitro. J Mol Biol 2003; 325: 913-35.

107. Bjornson KP, Moore KJ, Lohman TM. Kinetic mechanism of DNA binding and DNA-induced dimerization of the Escherichia coli Rep helicase. Biochemistry 1996; 35: 2268-82.

108. Rajendran S, Jezewska MJ, Bujalowski W. Multiple-step kinetic mechanism of DNA-independent ATP binding and hydrolysis by Escherichia coli replicative helicase DnaB protein: quantitative analysis using the rapid quench-flow method. J Mol Biol 2000; 303: 773-95.

109. Ellis DJ, Dryden DT, Berge T, Edwardson JM, Henderson RM. Direct observation of DNA translocation and cleavage by the EcoKI endonuclease using atomic force microscopy. Nat Struct Biol 1999; 6: 15-7.

110. Seidel R, van Noort J, van der Scheer C, Bloom JG, Dekker NH, Dutta CF, Blundell A, Robinson T, Firman K, Dekker C. Real-time observation of DNA translocation by the type I restriction modification enzyme EcoR124l. Nat Struct Mol Biol 2004; 11: 838-43.

111. Szczelkun MD. Kinetic models of translocation, head-on collision, and DNA cleavage by Type I restriction endonucleases. Biochemistry 2002; 41: 2067-74.

112. Seidel R, Bloom JG, Dekker C, Szczelkun MD. Motor step size and ATP coupling efficiency of the dsDNA translocase EcoR124l. EMBO J 2008; 27: 1388-98.

113. Feiss M, Catalano CE. Bacteriophage lambda terminase and the mechanisms of viral DNA packaging. In: Catalano CE, editor. Viral genome packaging machines: genetics, structure, and mechanism. Boston, MA: Springer US, 2005: 5-33.

114. Yang Q, Catalano CE. A minimal kinetic model for a viral DNA packaging machine. Biochemistry 2004; 43; 289-99.

115. Yang Q, Catalano CE, Maluf NK. Kinetic analysis of the genome packaging reaction in bacteriophage lambda. Biochemistry 2009; 48: 10705-15. 
116. Smith DE, Tans SJ, Smith SB, Grimes S, Anderson DL, Bustamante $C$. The bacteriophage straight phi29 portal motor can package DNA against a large internal force. Nature 2001; 413: 748-52.

117. Fuller DN, Rickgauer JP, Jardine PJ, Grimes S, Anderson DL, Smith DE. lonic effects on viral DNA packaging and portal motor function in bacteriophage phi 29. Proc Natl Acad Sci USA 2007; 104: 11245-50.

118. Rao VB, Feiss M. The Bacteriophage DNA Packaging Motor. 2008.

119. Barr FA, Silljé HH, Nigg EA. Polo-like kinases and the orchestration of cell division. Nat Rev Mol Cell Biol 2004; 5: 429-40.

120. Baumann C, Körner R, Hofmann K, Nigg EA. PICH, a centromere-associated SNF2 family ATPase, is regulated by Plk1 and required for the spindle checkpoint. Cell 2007; 128: 101-14.

121. Biebricher A, Hirano S, Enzlin JH, Wiechens N, Streicher WW, Huttner D, Wang LH, Nigg EA, Owen-Hughes T, Liu Y, Peterman E, Wuite GJ, Hickson ID. PICH: a DNA translocase specially adapted for processing anaphase bridge DNA. Mol Cell 2013; 51: 691-701.

122. Begg KJ, Dewar SJ, Donachie WD. A new Escherichia coli cell division gene, ftsK. J Bacteriol 1995; 177: 6211-22.

123. Aussel L, Barre FX, Aroyo M, Stasiak A, Stasiak AZ, Sherratt D. FtsK Is a DNA motor protein that activates chromosome dimer resolution by switching the catalytic state of the XerC and XerD recombinases. Cell 2002; 108: 195-205.

124. Cattoni DI, Chara O, Godefroy C, Margeat E, Trigueros S, Milhiet P-E, Nöllmann M. SpollIE mechanism of directional translocation involves target search coupled to sequencedependent motor stimulation. EMBO Rep 2013; 14: 473-9.

125. Saleh OA, Pérals C, Barre F-X, Allemand J-F. Fast, DNAsequence independent translocation by FtsK in a single-molecule experiment. EMBO J. 2004; 23: 2430-9.

126. Lee JY, Finkelstein IJ, Arciszewska LK, Sherratt DJ, Greene EC. Single-molecule imaging of FtsK translocation reveals mechanistic features of protein-protein collisions on DNA. Mol Cell 2014; 54: 832-43.

127. Pease PJ, Levy O, Cost GJ, Gore J, Ptacin JL, Sherratt D, Bustamante C, Cozzarelli NR. Sequence-directed DNA translocation by purified FtsK. Science 2005; 307: 586-90.

128. Levy O, Ptacin JL, Pease PJ, Gore J, Eisen MB, Bustamante C, Cozzarelli NR. Identification of oligonucleotide sequences that direct the movement of the Escherichia coli FtsK translocase. Proc Natl Acad Sci USA 2005; 102: 17618-23.
129. Bigot S, Saleh OA, Lesterlin C, Pages C, El Karoui M, Dennis C, Grigoriev M, Allemand J-F, Barre F-X, Cornet F. KOPS: DNA motifs that control E. coli chromosome segregation by orienting the FtsK translocase. EMBO J 2005; 24: 3770-80.

\section{Bionotes}

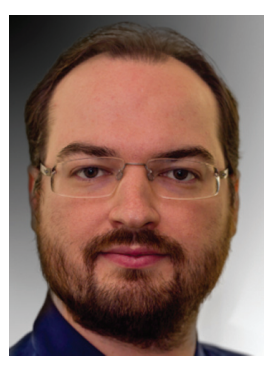

\section{Koan Briggs}

Department of Physics and Astronomy, University of Kansas, 1082 Malott Hall, 1251 Wescoe Hall Dr., Lawrence, KS 66045, USA

Koan Briggs is currently a graduate research assistant at the University of Kansas. He is pursuing a $\mathrm{PhD}$ in physics specializing in biophysics \& bioinformatics. Due to his background in mathematics and large scale computing his research activities are focused on understanding emergent statistical properties in biological systems, with an eye towards experimentally confirmable properties

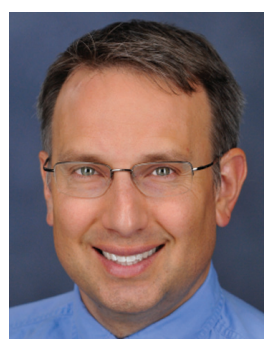

\section{Christopher J. Fischer}

Department of Physics and Astronomy, University of Kansas, 1082 Malott Hall, 1251 Wescoe Hall Dr., Lawrence, KS 66045, USA, shark@ku.edu

Christopher J. Fischer is an associate professor in the Department of Physics and Astronomy at the University of Kansas. He holds a PhD in applied physics from the University of Michigan and conducted postdoctoral research at Washington University School of Medicine. His biophysics research interests include the kinetics and thermodynamics of protein-protein and protein-nucleic acid interactions, and the development and implementation of new biophysical methodologies and techniques for the study of these phenomena. Recently his work has focused on understanding the function of molecular motors, especially those that manipulate DNA structure. 